\title{
Efectos del aislamiento en adultos mayores durante la pandemia de COVID-19: una revisión de la literatura
} Effects of isolation in older adults during COVID-19
pandemic: a review of the literature

Correspondencia Alexandra Granda-Oblitas alexandrago1997@gmail.com

Recibido: 27/10/2021 Arbitrado por pares Aprobado: 27/12/2021

Citar como: Granda-Oblitas A Quiroz-Gil GX, Runzer-Colmenares $F M$. Efectos del aislamiento en adultos mayores durante la pandemia de COVID-19: una revisión de la literatura. Acta Med Peru. 2021;38(4): 305-12. doi: https://doi. org/10.35663/amp.2021.384.2225

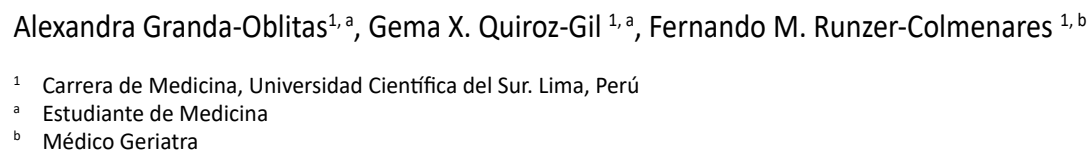

\section{RESUMEN}

El aislamiento como medida restrictiva durante la pandemia por el virus SARS-COV-2 ha generado efectos negativos en la población, especialmente en la salud de los adultos mayores. Se realizó una revisión de la literatura empleando las bases de datos de SCOPUS, Google Scholar, PubMed y Scielo publicados a partir de diciembre de 2019. Para los resultados, se hizo la asociación de aislamiento social con COVID-19, consecuencias físicas, efectos psicológicos, enfermedades neurodegenerativas, y la evaluación y manejo. Los artículos empleados brindan información de un periodo de tiempo determinado, por lo cual se desconoce cuáles podrían ser los efectos a largo plazo, y si realmente hay una diferencia con el estado de salud previo a la pandemia. Sin embargo, es de suma importancia establecer medidas para mitigar los efectos tanto a nivel físico como mental, que estén adaptadas a la realidad de cada país.

Palabras clave: Aislamiento Social, COVID-19, Salud Mental, Pandemias, Soledad (fuente: DeCSBIREME)

\section{ABSTRACT}

Isolation as a restrictive measure during the SARS-COV-2 pandemic has generated negative effects on the population, especially on the health of older adults. A literature review was conducted using SCOPUS, Google Scholar, PubMed and Scielo databases published as of December 2019. For the results, the association of social isolation with COVID-19, physical consequences, psychological effects, neurodegenerative diseases, and assessment and management was made. The articles used provide information from a specific time period, so it is unknown what the long-term effects might be, and whether there is really a difference with the pre-pandemic health status. However, it is of utmost importance to establish measures to mitigate the effects both physically and mentally, that are adapted to the reality of each country.

Key words: Social Isolation, COVID-19, Mental Health, Pandemics, Loneliness (source: MeSH NLM) 


\section{INTRODUCCIÓN}

En diciembre del 2019 se identificaron casos de infección respiratoria aguda por el virus SARS-Cov-2, y no mucho tiempo después estábamos frente a una pandemia que lo cambiaría todo. Aquello motivó a tomar medidas sanitarias en base a las recomendaciones de la Organización Mundial de la Salud (OMS), donde se le dio mayor importancia al aislamiento social y el distanciamiento, así como el lavado de manos, el uso de mascarillas, entre otras restricciones ${ }^{[1]}$. La enfermedad por coronavirus (COVID-19) afectó de distintas formas a cada grupo etario, exponiendo a los adultos mayores como uno de los grupos más vulnerables. Es muy conocido que el contacto y las actividades en comunidad, son necesarias para las relaciones interpersonales, así como para la salud física y mental ${ }^{[2]}$. Por ello, es razonable preocuparse por los efectos que podrían generarse a largo plazo, en un paciente complejo y vulnerable como lo es el adulto mayor ${ }^{[3]}$.

En cuanto a la salud mental, diversos estudios han demostrado que existe un mayor riesgo en el incremento de casos de ansiedad, depresión, alteración del sueño, irritabilidad, estrés, así como la exacerbación de enfermedades mentales preexistentes ${ }^{[4-6]}$. Asimismo, se ha reportado una mayor incidencia de suicidios, donde se identificaron como principales causas el miedo al contagio, el sentimiento de soledad, el abandono social y la desesperanza ${ }^{[7,8]}$. Debemos tener en cuenta que esta población en particular se caracteriza por ser más propensa a tener múltiples comorbilidades, que implican la necesidad de un monitoreo constante, el cual por el contexto de la pandemia no ha sido factible ${ }^{[9]}$. Dado que se priorizaron emergencias y casos por la COVID-19, se tuvo que reemplazar la consulta ambulatoria por la telemedicina, que si bien ha tenido un impacto positivo no tuvo el alcance esperado ${ }^{[10,11]}$

Es debido a lo antes mencionado, que los efectos generados por el aislamiento social sobre la salud pueden haber incrementado el riesgo de caídas, deterioro cognitivo, fragilidad y la carencia de cuidados ${ }^{[12]}$. Como resultado, diversos países optaron por emplear estrategias para contrarrestar las consecuencias desencadenadas por las medidas sanitarias impuestas ${ }^{[13-15]}$. Estas se basaron principalmente en incentivar el uso de diferentes dispositivos tecnológicos para aminorar la sensación de soledad ${ }^{[16-18]}$. De esta manera, se pusieron a disposición mayores alternativas para la interacción con otras personas, haciendo empleo de redes sociales, videollamadas y clases grupales ${ }^{[19,20]}$. También se promovieron las actividades de ocio, donde se hace especial énfasis en las actividades físicas que ayuden a evitar el sedentarismo en casa ${ }^{[2]}$.

El objetivo de este estudio es analizar toda la información disponible sobre los efectos del aislamiento social en adultos mayores durante la pandemia por COVID-19. A la vez, se brindará información sintetizada con estrategias satisfactorias que puedan ayudar a crear políticas o guías para contrarrestar el impacto negativo que puede ocasionar una pandemia sobre el adulto mayor.

\section{METODOLOGÍA}

Se realizó una búsqueda bibliográfica empleando las bases de datos de Pubmed, Scielo, Google Scholar, Embase y Scopus. Para obtener información más relevante sobre el tema, se utilizaron términos DECS (adulto mayor, aislamiento social, COVID-19, salud mental y pandemia) además de MeSH (elderly, older adults, social isolation, quarantine, loneliness, social-exclusion, pandemic, COVID-19, SARS-Cov-2 y mental health) con la ayuda de operadores booleanos. Se incluyeron artículos de cualquier diseño metodológico publicados a partir del año 2019 en adelante, tanto en español como en inglés. Por otro lado, se excluyeron aquellos artículos que no se encontraban disponibles gratuitamente o estuvieran incompletos, así como cartas al editor y aquellos estudios que tuvieran un tamaño muestral no justificado mediante un cálculo adecuado. Asimismo, se eliminaron las investigaciones que no incluyeran adultos mayores como objeto de estudio. Los artículos fueron descargados e ingresados al gestor bibliográfico Zotero, con el cual se facilitó la división en subtemas y se pudo evitar la duplicación de documentos en la base de datos.

\section{RESULTADOS}

\section{Resultados de la búsqueda}

Tras la filtración manual y a través de las bases de datos mencionadas anteriormente, se obtuvo un total de 1116 artículos: 553 en Pubmed, 220 en Google Scholar, 219 en Scopus, cinco de Scielo y 119 de Embase. Se descartaron los estudios que no cumplían con los criterios de selección y aquellos que se encontraban duplicados en más de una base de datos.

Luego de emplear los algoritmos de búsqueda se identificó un total de 155 artículos (69 en Pubmed, 38 en Scopus, 42 en Google Scholar, de Scielo, dos de Embase), que incluían participantes de continentes como América, Asia y Europa. Todos los estudios fueron descriptivos.

\section{Aislamiento social y COVID-19}

Conforme la esperanza de vida de la población mundial aumenta, se observa un incremento de adultos mayores viviendo solos, lo cual se convierte en un factor contribuyente para la epidemia de aislamiento social, que nació mucho antes que el distanciamiento físico implementado.

Se ha comprobado que el aislamiento social tiene un impacto negativo sobre la salud de las personas, y más aún cuando este es prolongado. Generalmente, los adultos mayores son quienes más se ven afectados debido a que presentan diversas comorbilidades, y por ello representan un grupo vulnerable. Cabe resaltar que la misma pandemia condicionó a que se retrasara 0 disminuyera la atención prestada por parte de las entidades de salud. En consecuencia, se dio un mal manejo de enfermedades 
crónicas tanto físicas como mentales y la ausencia de diagnósticos oportunos.

Actualmente, se desconoce cuáles podrían ser los efectos a largo plazo, pero se han identificado variables que podrían intervenir como la calidad de vida, sus creencias, independencia y la preocupación por su salud. En los artículos revisados se identificó una variedad de repercusiones tanto a nivel físico, mental y social durante el aislamiento, que se expondrán a lo largo de esta revisión.

\section{Aislamiento social y consecuencias físicas}

En la pandemia por el virus SARS-Cov-2, se ha podido comprobar que existe una asociación entre el aislamiento social y la inactividad física ${ }^{[21]}$. Diversos estudios respaldan que la participación social en actividades físicas ayuda a fortalecer los músculos, mejorar la función pulmonar, y también disminuyen las discapacidades, inflamación crónica, estrés oxidativo y los síndromes geriátricos en general ${ }^{[22]}$. En consecuencia, son preocupantes los efectos que podrían generarse a partir de un estilo de vida sin esta participación en actividades que promuevan el ejercicio.

Las medidas de confinamiento por COVID-19, llevaron a que los adultos mayores adopten una vida sedentaria, lo cual afectó de manera negativa sobre su salud física ${ }^{[23]}$. Algunos de los efectos se representaron como mayores enfermedades cardiovasculares, accidentes cerebrovasculares, un declive cognitivo, desórdenes musculoesqueléticos, y en general un aumento en la morbimortalidad, que se agravó con el sentimiento de soledad del individuo ${ }^{[14]}$. Uno de los mayores cambios identificados se vio reflejado en la dieta, la cual era poco saludable y escasa ${ }^{[12]}$. También, se evidenció un incremento en el consumo de alcohol y tabaco. Sin embargo, hubo estudios en los que sí se mantuvo una dieta balanceada y hábitos saludables, pero fueron representados por una minoría.

De igual modo, se ha comprobado que la reducción de actividades físicas a corto plazo puede afectar la sensibilidad a la insulina, la función cardiorrespiratoria, y además aumentar el porcentaje de grasa visceral y el desarrollo de dislipidemia. Aquello, sumado a los periodos de inactividad generados por el cambio de estilo de vida tras la pandemia por COVID-19, pueden provocar una crisis catabólica que en consecuencia aumentaría el riesgo de caídas, fracturas y discapacidades. Adicionalmente, esta carencia parcial o total de actividad física se ha visto asociada a una disminución en la masa muscular, y cabe resaltar que los adultos mayores pierden fisiológicamente un aproximado de $0,8 \%$ de masa muscular al año, lo cual podría deteriorar aún más su salud ${ }^{[23]}$.

Por otra parte, se conoce que el sueño ha sido una de las principales funciones biológicas vulneradas ${ }^{[24]}$. Debido al aislamiento se ha visto afectado el ciclo circadiano, ya que eventos estresantes pueden generar la alteración de este, así como la limitada exposición a la luz del día y la ausencia de rutinas diarias ${ }^{[25]}$. Por ende, la disminución del sueño resulta en una secreción inadecuada de melatonina, la cual está asociada a cambios en la función inmunitaria y a procesos fibróticos en hígado, riñón y sistema vascular, haciéndolo más susceptible a una infección por el virus SARS-Cov-2 ${ }^{[26]}$.

\section{Aislamiento social y efectos psicológicos}

Según la OMS, aproximadamente el $7 \%$ de los adultos mayores tiene un trastorno depresivo mayor y el 3,8 \% un trastorno de ansiedad ${ }^{[27]}$. Asimismo, establecen que aquellos que se encuentran en aislamiento social o con deterioro cognitivo pueden volverse aún más ansiosos, enojados, estresados, agitados y retraídos durante las medidas restrictivas por la pandemia ${ }^{[1,28]}$.

Debido a esto, se han incrementado los problemas de salud mental, que abarcan desde depresión, ansiedad, insomnio, sentimiento de soledad, fobias, estrés post traumático, autolesiones y hasta intentos de suicidio ${ }^{[9]}$. Existen diversos factores que han contribuido a agravar esta problemática. Por ejemplo, la difusión de información alarmista y poco veraz por parte de los medios de comunicación, sumado a la incertidumbre social, política y económica, el miedo a contagiarse o de perder a un ser querido. Asimismo, la gestión de los gobiernos y respuesta frente a la pandemia fueron factores determinantes, ya que la percepción de menor apoyo se reflejaba en mayores síntomas de depresión y ansiedad ${ }^{[29-31]}$.

Por otro lado, se vieron comprometidas las necesidades de cuidado básico como: la limpieza, alimentación y el acceso a los servicios de salud mental. Este último generó mayor dificultad para la ejecución de tratamientos, seguimiento de enfermedades psiquiátricas severas y terapias como la electroconvulsiva ${ }^{[32-35]}$.

Estas variables sumadas a diversos estereotipos y creencias que se ven intensificadas por la misma sociedad e incluso autoridades, que muchas veces consideran a los adultos mayores como una carga social, conllevan a la discriminación por edad, denominada edadismo. En consecuencia, esta provoca exacerbaciones del estrés, ansiedad y/o depresión en los adultos mayores ${ }^{[36-40]}$.

Múltiples estudios han demostrado que, tras la pandemia, existe una mayor susceptibilidad hacia síntomas psiquiátricos (depresivos, ansiosos, de estrés postraumático, entre otros) en las mujeres en comparación con los hombres ${ }^{[3,41]}$. También se halló una asociación entre el lugar de residencia como las zonas urbanas, y la presencia de ansiedad o pensamientos rumiantes como factores predictivos de insomnio, ya que provocan una excitación cognitiva, lo que puede alterar la homeostasis del cortisol, dando como resultado un sueño insuficiente ${ }^{[9]}$. Asimismo, se identificó que aquellos que presentaban mayor fobia hacia la COVID-19, eran precisamente mujeres, solteras, que vivían solas y tenían un nivel socioeconómico más alto ${ }^{[42]}$.

En contraposición, se ha demostrado que los hombres pueden tener mayor riesgo de reportar empeoramiento de depresión y ansiedad después del encierro, aún más si estos son solteros, 
viudos, divorciados o viven solos. Una posible hipótesis establece que puede deberse a la tendencia de los hombres a ser reacios a admitir sus miedos y la dificultad que pueden presentar para expresar sus emociones y buscar apoyo ${ }^{[9,43]}$.

Adicionalmente a los factores inherentes del género y estado civil, se ha visto que las comorbilidades preexistentes pueden verse incrementadas por la fragilidad del adulto mayor, que es traducida como una respuesta fisiológica inadecuada ante eventos estresantes, que podrían hacerlos más susceptibles a caídas y/o discapacidades. Sumado a ello, el aislamiento social al que se enfrentan genera un sentimiento de soledad y abandono, volviéndolo más propenso a un estado de vulnerabilidad, lo que sustenta la teoría sobre la etiopatogenia del trastorno depresivo mayor al final de la vida ${ }^{[9,43-45]}$. Al mismo tiempo, existen estudios que corroboran que un alto sentimiento de soledad está asociado a una disminución del apetito, mayor consumo de comida rápida, lo cual conlleva a malnutrición, déficit de ácido fólico, vitamina B12, y antioxidantes, que en consecuencia contribuyen a mayores síntomas depresivos ${ }^{[46,47]}$.

Si bien los adultos mayores son considerados un grupo vulnerable para diversas patologías psiquiátricas, varias investigaciones han demostrado que son los que mejor respuesta tienen frente a episodios de ansiedad y depresión en comparación con la población joven. Algunas justificaciones se basan en que presentan mayor resiliencia, regulación de sus emociones mediante la aceptación, reinterpretación, distracción o supresión ${ }^{[31,48]}$. Sin embargo, aún se carece de estudios que corroboren cuáles podrían ser las diferencias entre un antes y durante de una pandemia y los posibles efectos a largo plazo ${ }^{[6,49]}$. Es importante recalcar que el grado de resiliencia podría variar en base a factores internos (respuesta biológica al estrés, personalidad, salud física, capacidad cognitiva, etc.) y externos (estabilidad financiera, estatus social, conexión con sus familiares, tecnología, entre otros) ${ }^{[15,50]}$. Por ejemplo, una población estudiada en Reino Unido no presentó mayor cambio en cuanto a síntomas psiquiátricos, ya que anteriormente habían experimentado situaciones semejantes de aislamiento social por la Segunda Guerra Mundial y las pandemias de influenza en 1957 y $1968^{\text {[51] }}$.A pesar de que algunos tuvieron una mejor respuesta, otros no obtuvieron el mismo resultado. La pandemia por COVID-19 provocó el incremento del riesgo de "muertes por desesperación" y exacerbó los riesgos preexistentes de suicidio en la vejez, aún más en personas con trastornos afectivos ${ }^{[28,52]}$. En la actualidad se desconoce realmente si la pandemia ha afectado la tasa de suicidios. No obstante, esta puede resultar en la confluencia de factores de riesgo como vivir solo, la soledad y el aislamiento social para comportamientos suicidas ${ }^{[53]}$.

\section{Aislamiento social y enfermedades neurodegenerativas}

En cuanto a los adultos mayores con demencia, estos se encuentran en un estado de mayor vulnerabilidad por síntomas como la amnesia, apraxia, agnosia e incapacidad ejecutiva. Los cuales conllevan a cometer más errores y a tener una menor adherencia hacia las medidas sanitarias (lavado de manos, uso de mascarillas, distanciamiento social) frente a la pandemia por COVID-19 ${ }^{[54]}$.

Asimismo, estas medidas implican un cambio en sus rutinas, lo cual provoca una carga cognitiva adicional, que puede verse reflejada en una disminución de la concentración, memoria, orientación y comunicación, así como el empeoramiento y surgimiento de síntomas neuropsiquiátricos (cambios de humor, apatía, agitación motora, agresión, ansiedad, ideación delirante, insomnio, confusión creciente y depresión) ${ }^{[54-58]}$. Esto generó un incremento del uso de fármacos psicotrópicos (hipnóticos antipsicóticos, benzodiacepinas y antidepresivos) independientemente del grado de demencia. Específicamente en pacientes con Alzheimer, hay estudios donde se ha observado el aumento de estos síntomas, siendo la ansiedad el más frecuente ${ }^{[7,55]}$. Además, esta clínica fue más evidente en casos de demencia leve en comparación con aquellos casos más avanzados. Una posible explicación es que los más leves podrían haber sufrido cambios más radicales en su estilo de vida durante la pandemia, y eran más conscientes del contexto en el que vivían. En cambio, en los más graves no hubo mucha variación, ya que por lo general se encuentran confinados y son menos activos. De igual manera, se vieron comprometidas las medidas que normalmente se utilizaban para contrarrestar los trastornos del sueño y de la marcha, puesto que el aislamiento social conlleva a una disminución de la realización de actividades físicas y rutinas estructuradas. En consecuencia, se genera un mayor riesgo de caídas y disminución en la calidad de vida, convirtiéndolo en un adulto mayor frágil ${ }^{[7]}$.

En el caso de los pacientes con Parkinson, se afectaron en su mayoría los síntomas no motores (trastornos del sueño, ansiedad, depresión y trastornos cognitivos). Presentando un mayor deterioro en aquellos con depresión e insomnio preexistente, siendo las mujeres y jóvenes los más afectados ${ }^{[59]}$. Asimismo, el estrés psicológico generado por los brotes de la COVID-19 agravaron temporalmente los síntomas motores (temblores, congelación de la marcha o trastornos del movimiento). Otros estudios adicionales sugieren que, en pacientes con enfermedad por priones, la infección por la COVID-19 podría agravar las reacciones inflamatorias del cerebro y de esa forma acelerar la progresión de la enfermedad. También, añaden que el diagnóstico de Esclerosis Lateral Amiotrófica, así como otras enfermedades neurodegenerativas se han dificultado aún más por el contexto actual ${ }^{[57]}$.

\section{Evaluación y manejo: un reto a ser enfocado de forma holística}

A raíz de las medidas tomadas por los gobiernos, diversas organizaciones de la salud optaron por brindar recomendaciones básicas para sobrellevar el aislamiento social. Por ejemplo, la Organización Panamericana de la Salud (OPS) y la OMS propusieron que los adultos mayores debían recibir apoyo emocional, mantener conexiones sociales, una rutina, actividades físicas, además debía asegurarse la provisión de necesidades básicas y medicamentos e incentivar las actividades al aire libre 
con el debido distanciamiento ${ }^{[1,2,22]}$. Adicionalmente, brindar información veraz y comprensible sobre la enfermedad, para que incluso un paciente con deterioro cognitivo sea capaz de entenderla.

En una revisión realizada por la OMS se demostró que la participación en actividades artísticas puede mejorar el bienestar emocional de un individuo. Incluso escuchar música puede mejorar el sueño, la recuperación de pacientes con ictus y tener un impacto positivo sobre la demencia ${ }^{[60,61]}$. Cabe resaltar que las recomendaciones brindadas debían servir como base para la aplicación de medidas y soluciones específicas en cada país ${ }^{[62]}$. En el caso de Wuhan, China, se optó por una solución interdisciplinaria efectiva con la ayuda de los profesionales de la salud mental, trabajadores sociales, administradores de residencias de ancianos y voluntarios. También, ofrecieron un manual de autoayuda psicosocial, consejerías y guías específicas para el soporte de pacientes con deterioro cognitivo. Garantizaron un entorno seguro, proporcionando la provisión de equipos de protección personal, además les enseñaron cómo solicitar una cita médica virtual y contactar a un psiquiatra geriátrico cada vez que alguno lo requería ${ }^{[10]}$.

Otras medidas que han resultado efectivas en diversos países para hacer frente al aislamiento social y la soledad son el uso de redes sociales, videollamadas, aplicaciones móviles y telemedicina ${ }^{[19,63-65]}$. Además, programas de voluntarios dispuestos a proveer apoyo y compañía, visitadores médicos, el servicio de terapia cognitiva conductual, consejería y monitoreo online para una intervención psicológica fueron de gran ayuda para contrarrestar los efectos de la pandemia en la población mayor ${ }^{[11,66]}$.

Mientras algunos gobiernos se rigen únicamente por seguir estas recomendaciones, existen otros que buscan innovar implementando nuevas tecnologías o actualizando las preexistentes. Una de estas estrategias, que sigue actualmente en investigación, son los robots de telepresencia móvil que consisten en herramientas equipadas con cámara, un monitor, altavoz y cuerpo con ruedas que permite el movimiento, con el fin de poder comunicar de una manera más interactiva a dos individuos que se encuentren lejos ${ }^{[67]}$. Se ha planteado que podrían utilizarse no sólo para comunicarse con familiares sino también como parte de la telemedicina. También está el Audrey- Flower, un robot de asistencia social que tiene como objetivo reducir el aburrimiento y soledad mediante la estimulación intelectual a través de juegos, que facilitaría las llamadas y fomentaría un estilo de vida saludable, además de la detección temprana de accidentes cerebrovasculares y caídas para obtener ayuda en emergencias ${ }^{[68]}$.

Si bien hay estudios que demuestran su aceptación y utilidad, hay que tener en cuenta factores económicos, el riesgo de deshumanización y también la capacitación que conlleva. En general, el uso de la tecnología tiene una variedad de limitaciones para los adultos mayores, como factores personales del individuo (edad, género, educación, ingresos, estado salud, experiencia previa en tecnología), relevancia social, contextos nacionales, entre otros (utilidad, simplicidad percibida, asequibilidad, accesibilidad, apoyo técnico y social, independencia, experiencia y confianza) ${ }^{[66,69]}$. Por ello, centrarse solo en soluciones digitales, podría potencialmente perpetuar la discriminación por edad ${ }^{[70]}$.

\section{CONCLUSIONES}

En conclusión, los estudios revisados sugieren que el aislamiento social por la pandemia del virus SARS-Cov-2 ha tenido un impacto negativo sobre la salud de los adultos mayores y ha contribuido a la discriminación del mismo. No obstante, se desconocen cuáles podrían ser los efectos a largo plazo.

Dentro de las repercusiones a nivel mental se ha apreciado un aumento del estrés, ansiedad, soledad, alteración del sueño, depresión e incluso algunos casos de suicidios. Específicamente, aquellos pacientes con enfermedades neurodegenerativas mostraron ser más vulnerables por su condición física y mental preexistente. Asimismo, un incremento en el estilo de vida sedentario y hábitos nocivos, conllevaron a un incremento de factores de riesgo para las comorbilidades.

En consecuencia, organizaciones como la OMS y OPS propusieron recomendaciones interdisciplinarias con el fin de mitigar los efectos del aislamiento social. Sin embargo, cada gobierno adaptó estas medidas según el contexto y realidad de su país, siendo el uso de la tecnología una de las más relevantes, con el cual se ha encontrado múltiples beneficios, así como limitaciones.

Contribución de los autores: Los tres autores plasmaron la idea de investigación, se encargaron de la búsqueda de información, participaron en la redacción e interpretación de resultados y aprobaron la revisión final del documento.

Potenciales conflictos de intereses: Los autores niegan algún conflicto de interés.

Fuentes de financiamiento: Autofinanciado.

\section{ORCID}

Alexandra Granda-Oblitas: https://orcid.org/0000-0002-29159002,

Gema X. Quiroz-Gil: https://orcid.org/0000-0001-9543-2049

Fernando M. Runzer-Colmenares: https://orcid.org/0000-00034045-0260

\section{REFERENCIAS BIBLIOGRÁFICAS}

1. Organización Panamericana de la Salud. Consideraciones psicosociales y de salud mental durante el brote de COVID-19 [Internet]. 2020 [citado 28 de junio de 2021]. Disponible en: https:// www.paho.org/sites/default/files/2020-03/smaps-coronavirus-esfinal-17-mar-20.pdf 
2. Hwang T-J, Rabheru K, Peisah C, Reichman W, Ikeda M. Loneliness and social isolation during the COVID-19 pandemic. International Psychogeriatrics. octubre de 2020;32(10):1217-20. doi: 10.1017/ S1041610220000988

3. Romero DE, Muzy J, Damacena GN, Souza NA de, Almeida W da S de, Szwarcwald CL, et al. Older adults in the context of the COVID-19 pandemic in Brazil: effects on health, income and work. Cad Saude Publica. 2021;37(3). doi: 10.1590/0102-311X00216620

4. Roy J, Jain R, Golamari R, Vunnam R, Sahu N. COVID-19 in the geriatric population. Int J Geriatr Psychiatry. diciembre de 2020;35(12):1437-41. doi: 10.1002/gps.5389

5. Broche-Pérez Y, Fernández-Castillo E, Reyes Luzardo DA. Consecuencias psicológicas de la cuarentena y el aislamiento social durante la pandemia de COVID-19. Rev cub salud pública. 5 de febrero de 2021;46:e2488.

6. Fujita $\mathrm{K}$, Inoue A, Kuzuya $\mathrm{M}$, Uno $\mathrm{C}$, Huang $\mathrm{CH}$, Umegaki $\mathrm{H}$, et al. Mental health status of the older adults in japan during the COVID-19 pandemic. J Am Med Dir Assoc. enero de 2021;22(1):2201. doi: $10.1016 /$ j.jamda.2020.11.023

7. Cohen G, Russo MJ, Campos JA, Allegri RF. COVID-19 epidemic in Argentina: Worsening of behavioral symptoms in elderly subjects with dementia living in the community. Front Psychiatry. 28 de agosto de 2020;11:866. doi: 10.3389/fpsyt.2020.00866

8. Frenkel-Yosef M, Maytles R, Shrira A. Loneliness and its concomitants among older adults during the COVID-19 pandemic. Int Psychogeriatr. octubre de 2020;32(10):1257-9. doi: 10.1017/ S1041610220003476

9. Robb CE, de Jager CA, Ahmadi-Abhari S, Giannakopoulou P, UdehMomoh C, McKeand J, et al. Associations of social isolation with anxiety and depression during the early COVID-19 pandemic: $A$ survey of older adults in london, uk. front psychiatry [Internet]. 17 de septiembre de 2020 [citado 19 de febrero de 2021];11. Disponible en: https://www.ncbi.nlm.nih.gov/pmc/articles/ PMC7566017/ doi: 10.3389/fpsyt.2020.591120

10. Wang H, Li T, Gauthier S, Yu E, Tang Y, Barbarino P, et al. Coronavirus epidemic and geriatric mental healthcare in China: How a coordinated response by professional organizations helped older adults during an unprecedented crisis. Int Psychogeriatr. octubre de 2020;32(10):1117-20. doi: 10.1017/S1041610220000551

11. Batra K, Morgan AE, Sharma M. COVID-19 and social isolation endangering psychological health of older adults: Implications for telepsychiatry. Signa Vitae. 2020;16(2):14-9. doi: 10.22514/ sv.2020.16.0070

12. Lebrasseur A, Fortin-Bédard N, Lettre J, Raymond E, Bussières E-L, Lapierre N, et al. Impact of the COVID-19 pandemic on older adults: Rapid review. JMIR Aging. 12 de abril de 2021;4(2):e26474. doi: $10.2196 / 26474$

13. Lithander FE, Neumann S, Tenison E, Lloyd K, Welsh TJ, Rodrigues $\mathrm{JCL}$, et al. COVID-19 in older people: a rapid clinical review. Age and Ageing. 1 de julio de 2020;49(4):501-15. doi: 10.1093/ageing/ afaa093

14. Wickens CM, McDonald AJ, Elton-Marshall T, Wells S, Nigatu YT, Jankowicz D, et al. Loneliness in the COVID-19 pandemic: Associations with age, gender and their interaction. J Psychiatr Res. abril de 2021;136:103-8. doi: 10.1016/j.jpsychires.2021.01.047

15. Bastoni S, Wrede C, Ammar A, Braakman-Jansen A, Sanderman R, Gaggioli A, et al. Psychosocial Effects and Use of Communication Technologies during Home Confinement in the First Wave of the COVID-19 Pandemic in Italy and The Netherlands. Int J Environ Res Public Health. 5 de marzo de 2021;18(5). doi: 10.3390/ ijerph18052619
16. Hebblethwaite S, Young L, Rubio TM. Pandemic precarity: Aging and social engagement. Leisure Sciences. 1 de marzo de 2021;43(12):170-6. doi: 10.1080/01490400.2020.1773998

17. Xie B, Charness N, Fingerman K, Kaye J, Kim MT, Khurshid A. When going digital becomes a necessity: Ensuring older adults' needs for information, services, and social inclusion during COVID-19. Journal of Aging \& Social Policy. 3 de julio de 2020;32(4-5):460-70. doi: $10.1080 / 08959420.2020 .1771237$

18. Wu B. Social isolation and loneliness among older adults in the context of COVID-19: A global challenge. Glob Health Res Policy. 2020;5:27. doi: 10.1186/s41256-020-00154-3

19. Conroy KM, Krishnan S, Mittelstaedt S, Patel SS. Technological advancements to address elderly loneliness: practical considerations and community resilience implications for COVID-19 pandemic. Working with Older People. 1 de enero de 2020;24(4):257-64. doi: 10.1108/wwop-07-2020-0036

20. Goulabchand R, Boclé $H$, Vignet R, Sotto A, Loubet P. Digital tablets to improve quality of life of COVID-19 older inpatients during lockdown. European Geriatric Medicine. 2020;11(4):705-6. doi: 10.1007/s41999-020-00344-9

21. Sepúlveda-Loyola W, Rodríguez-Sánchez I, Pérez-Rodríguez P, Ganz F, Torralba R, Oliveira DV, et al. Impact of social isolation due to covid-19 on health in older people: Mental and physical effects and recommendations. J Nutr Health Aging. 2020;24(9):938-47. doi: 10.1007/s12603-020-1500-7

22. Machado CLF, Pinto RS, Brusco CM, Cadore EL, Radaelli R. COVID-19 pandemic is an urgent time for older people to practice resistance exercise at home. Exp Gerontol. noviembre de 2020;141. doi: 10.1016/j.exger.2020.111101

23. Moro T, Paoli A. When COVID-19 affects muscle: Effects of quarantine in older adults. European Journal of Translational Myology. 2020;30(2):219-22. doi: 10.4081/ejtm.2019.9069

24. Lozupone M, La Montagna M, Di Gioia I, Sardone R, Resta E, Daniele $A$, et al. Social frailty in the COVID-19 pandemic era. Front Psychiatry. 3 de noviembre de 2020;11:577113. doi: 10.3389/ fpsyt.2020.577113

25. Cardinali DP, Brown GM, Reiter RJ, Pandi-Perumal SR. Elderly as a high-risk group during COVID-19 pandemic: Effect of circadian misalignment, sleep dysregulation and melatonin administration. Sleep Vigil. 26 de septiembre de 2020;1-7. doi: 10.1007/s41782020-00111-7

26. Damiot A, Pinto AJ, Turner JE, Gualano B. Immunological implications of physical inactivity among older adults during the COVID-19 pandemic. GER. 2020;66(5):431-8. doi: 10.1159/000509216

27. Organización Mundial de la Salud. La salud mental y los adultos mayores [Internet]. 2017 [citado 30 de junio de 2021]. Disponible en: https://www.who.int/es/news-room/fact-sheets/detail/lasalud-mental-y-los-adultos-mayores

28. Troutman-Jordan M, Kazemi DM. COVID-19's impact on the mental health of older adults: Increase in isolation, depression, and suicide risk. An urgent call for action. Public Health Nursing. 2020;37(5):637-8. doi: 10.1111/phn.12774

29. Callís S, Guarton OM, Cruz V, de Armas AM. Manifestaciones psicológicas en adultos mayores en aislamiento social durante la pandemia COVID-19. En: Primera Jornada Virtual de Psiquiatría 2020 [Internet]. 2020 [citado 21 de abril de 2021]. Disponible en: http://jvirtualpsiquiatria2020.sld.cu/index.php/jvpsq/2020/paper/ view/4

30. Yildirim H, Işik K, Aylaz R. The effect of anxiety levels of elderly people in quarantine on depression during covid-19 pandemic. Social Work in Public Health. 17 de febrero de 2021;36(2):194-204. Doi:10.1080/19371918.2020.1868372 
31. Röhr S, Reininghaus U, Riedel-Heller SG. Mental wellbeing in the German old age population largely unaltered during COVID-19 lockdown: results of a representative survey. BMC Geriatr. 23 de noviembre de 2020;20(1):489. doi: 10.1186/s12877-020-01889-x

32. Burlacu A, Mavrichi I, Crisan-Dabija R, Jugrin D, Buju S, Artene B, et al. "Celebrating old age»: an obsolete expression during the COVID-19 pandemic? Medical, social, psychological, and religious consequences of home isolation and loneliness among the elderly. Arch Med Sci. 2021;17(2):285-95. Doi:10.5114/aoms.2020.95955

33. Reppas-Rindlisbacher C, Finlay JM, Mahar AL, Siddhpuria S, Hallet J, Rochon PA, et al. Worries, attitudes, and mental health of older adults during the COVID-19 pandemic: Canadian and U.S. perspectives. Journal of the American Geriatrics Society. 2021;69(5):1147-54. Doi: 10.1111/jgs.17105

34. Khoury R, Karam G. Impact of COVID-19 on mental healthcare of older adults: insights from Lebanon (Middle East). Int Psychogeriatr. octubre de2020;32(10):1177-80. Doi:10.1017/ S104161022000068X

35. Wong SYS, Zhang D, Sit RWS, Yip BHK, Chung RY-N, Wong CKM, et al. Impact of COVID-19 on loneliness, mental health, and health service utilisation: a prospective cohort study of older adults with multimorbidity in primary care. Br J Gen Pract. noviembre de 2020;70(700):e817-24. Doi: 10.3399/bjgp20X713021

36. Skoog I. COVID-19 and mental health among older people in Sweden. Int Psychogeriatr. octubre de 2020;32(10):1173-5. Doi: 10.1017/S104161022000143X

37. Ayalon L. There is nothing new under the sun: Ageism and intergenerational tension in the age of the COVID-19 outbreak. Int Psychogeriatr. octubre de 2020;32(10):1221-4. Doi: 10.1017/ S1041610220000575

38. Hernández J. Impacto de la COVID-19 sobre la salud mental de las personas. Medicentro Electrónica 2020;(2020);24(3):578-594.

39. D'cruz M, Banerjee D. 'An invisible human rights crisis': The marginalization of older adults during the COVID-19 pandemic - An advocacy review. Psychiatry Research. 1 de octubre de 2020;292:113369. Doi: 10.1016/j.psychres.2020.113369

40. Fraser S, Lagacé $M$, Bongué B, Ndeye N, Guyot J, Bechard L, et al. Ageism and COVID-19: what does our society's response say about us?. Age and Ageing. 24 de agosto de 2020;49(5):692-5. Doi: 10.1093/ageing/afaa097

41. Parlapani E, Holeva V, Nikopoulou VA, Sereslis K, Athanasiadou M, Godosidis A, et al. Intolerance of Uncertainty and Loneliness in Older Adults During the COVID-19 Pandemic. Front Psychiatry [Internet]. 2020 [citado 19 de febrero de 2021];11. Disponible en: https://www.frontiersin.org/articles/10.3389/fpsyt.2020.00842/ full Doi: $10.3389 /$ fpsyt.2020.00842

42. Cihan FG, Gökgöz Durmaz F. Evaluation of COVID-19 phobia and the feeling of loneliness in the geriatric age group. Int J Clin Pract. 11 de febrero de 2021; 2021 Jun;75(6):e14089. Doi: 10.1111/ijcp.14089

43. Abu Kamel AK, Alnazly EK. The impact of confinement on older Jordanian adults' mental distress during the COVID-19 pandemic: A web-based cross-sectional study. Perspectives in Psychiatric Care. 2021;10.1111/ppc.12798. Doi: 10.1016/j.psychres.2020.113369

44. Naeim M, Rezaeisharif A, Kamran A. COVID-19 has made the elderly Ionelier. Dement Geriatr Cogn Dis Extra. abril de 2021;11(1):26-8. Doi: 10.1159/000514181

45. Grolli RE, Mingoti MED, Bertollo AG, Luzardo AR, Quevedo J, Réus GZ, et al. Impact of COVID-19 in the mental health in elderly: Psychological and biological updates. Mol Neurobiol. mayo de 2021;58(5):1905-16. Doi: 10.1007/s12035-020-02249-x
46. Vitman Schorr A, Yehuda I, Tamir S. Loneliness, malnutrition and change in subjective age among older adults during COVID-19 pandemic. Int J Environ Res Public Health. 26 de diciembre de 2020;18(1). Doi: 10.3390/ijerph18010106

47. Schorr AV, Yehuda I, Tamir S. Ethnic differences in loneliness, depression, and malnutrition among older adults during COVID-19 quarantine. J Nutr Health Aging. 2021;25(3):311-7. Doi: 10.1007/ s12603-020-1540-z

48. Losada-Baltar A, Márquez-González M, Jiménez-Gonzalo L, PedrosoChaparro MDS, Gallego-Alberto L, Fernandes-Pires J. Differences in anxiety, sadness, loneliness and comorbid anxiety and sadness as a function of age and self-perceptions of aging during the lock-out period due to COVID-19. Revista Espanola de Geriatria y Gerontologia. 2020;55(5):272-8. Doi: 10.1016/j.regg.2020.05.005

49. Dahlberg L. Loneliness during the COVID-19 pandemic. Aging \& Mental Health. 3 de julio de 2021;25(7):1161-4. Doi: 10.1080/13607863.2021.1875195

50. Vahia IV, Jeste DV, Reynolds CF lii. Older Adults and the Mental Health Effects of COVID-19. JAMA - Journal of the American Medical Association. 2020;324(22):2253-4. Doi: 10.1001/jama.2020.21753

51. Brown L, Mossabir R, Harrison N, Brundle C, Smith J, Clegg A. Life in lockdown: A telephone survey to investigate the impact of COVID-19 lockdown measures on the lives of older people ( $\geq 75$ years). Age Ageing. 26 de febrero de 2021;50(2):341-6. Doi: 10.1093/ageing/afaa255

52. Sheffler JL, Joiner TE, Sachs-Ericsson NJ. The interpersonal and psychological impacts of COVID-19 on risk for late-life suicide. Gerontologist. 21 de enero de 2021;61(1):23-9. Doi: 10.1093/ geront/gnaa103

53. Wand APF, Zhong B-L, Chiu HFK, Draper B, Leo DD. COVID-19: The implications for suicide in older adults. International Psychogeriatrics. octubre de 2020;32(10):1225-30. Doi: 10.1017/ S1041610220000770

54. Schapira M, Schapira M. Impacto psicosocial de la pandemia por COVID-19 en adultos mayores con demencia y sus cuidadores. Revista Argentina de Salud Pública. octubre de 2020;12:4-4.

55. Suárez-González A, Rajagopalan J, Livingston G, Alladi S. The effect of Covid-19 isolation measures on the cognition and mental health of people living with dementia: A rapid systematic review of one year of evidence. medRxiv. 20 de marzo de 2021. doi: 10.1101/2021.03.17.21253805

56. Manca R, De Marco M, Venneri A. The impact of COVID-19 infection and enforced prolonged social isolation on neuropsychiatric symptoms in older adults with and without dementia: A review. Front Psychiatry [Internet]. 2020 [citado 30 de junio de 2021];11. Disponible en: https://www.frontiersin.org/articles/10.3389/ fpsyt.2020.585540/full.Doi: 10.3389/fpsyt.2020.585540

57. Hu C, Chen C, Dong X-P. Impact of COVID-19 pandemic on patients with neurodegenerative diseases. Front Aging Neurosci [Internet] 2021 [citado 30 de junio de 2021];13. Disponible en: https:// www.frontiersin.org/articles/10.3389/fnagi.2021.664965/full.doi: 10.3389/fnagi.2021.664965

58. Simonetti A, Pais C, Jones M, Cipriani MC, Janiri D, Monti L, et al. Neuropsychiatric symptoms in elderly with dementia during COVID-19 pandemic: Definition, treatment, and future directions Front Psychiatry [Internet]. 29 de septiembre de 2020 [citado 19 de febrero de 2021];11. Disponible en: https://www.ncbi.nlm.nih. gov/pmc/articles/PMC7550649/.doi: 10.3389/fpsyt.2020.579842

59. Janiri D, Petracca M, Moccia L, Tricoli L, Piano C, Bove F, et al. COVID-19 pandemic and psychiatric symptoms: The impact on Parkinson's disease in the elderly. Front Psychiatry. 2020;11:581144. Doi: $10.3389 /$ fpsyt.2020.581144 
60. Hansen P, Main C, Hartling L. Dance intervention affects social connections and body appreciation among older adults in the long term despite COVID-19 social isolation: A mixed methods pilot study. Frontiers in Psychology. 2021;12. Doi: 10.3389/ fpsyg.2021.635938

61. Day P, Gould J, Hazelby G. A public health approach to social isolation in the elderly. Journal of Community Nursing. 23 de junio de 2020;34.

62. Universidad de Lima. Cómo apoyar a los adultos mayores durante la pandemia | Universidad de Lima [Internet]. 2020 [citado 30 de junio de 2021]. Disponible en: https://www.ulima.edu.pe/entrevista/ carlos-orosco-24-04-2020

63. Daly JR, Depp C, Graham SA, Jeste DV, Kim H-C, Lee EE, et al. Health impacts of the stay-at-home order on community-dwelling older adults and how technologies may help: Focus group study. JMIR Aging. 22 de marzo de 2021;4(1). Doi: 10.2196/25779

64. Nicol GE, Piccirillo JF, Mulsant BH, Lenze EJ. Action at a distance: Geriatric research during a pandemic. Journal of the American Geriatrics Society. 2020;68(5):922-5. Doi: 10.1111/jgs.16443

65. Banskota S, Healy M, Goldberg EM. 15 smartphone apps for older adults to use while in isolation during the COVID-19 pandemic. West J Emerg Med. mayo de 2020;21(3):514-25. Doi: 10.5811/ westjem.2020.4.47372
66. Gorenko JA, Moran C, Flynn M, Dobson K, Konnert C. Social isolation and psychological distress among older adults related to COVID-19: A narrative review of remotely-delivered interventions and recommendations. Journal of Applied Gerontology. 2021;40(1):313. Doi: $10.1177 / 0733464820958550$

67. Isabet $B$, Pino $M$, Lewis $M$, Benveniste $S$, Rigaud $A-S$. Social telepresence robots: A narrative review of experiments involving older adults before and during the COVID-19 pandemic. Int J Environ Res Public Health. 30 de marzo de 2021;18(7):3597. Doi: 10.3390/ ijerph18073597

68. Liberman-Pincu E. Audrey- Flower-like social assistive robot: Taking care of older adults in times of social isolation during the Covid-19 Pandemic. En 2021. p. 613-4. Doi: 10.1145/3434074.3446962

69. Sacco G, Lléonart S, Simon R, Noublanche F, Annweiler C, TOVID Study Group. Communication technology preferences of hospitalized and institutionalized frail older adults during COVID-19 confinement: Cross-sectional survey study. JMIR Mhealth Uhealth. 18 de septiembre de 2020;8(9):e21845. Doi: 10.2196/21845

70. Seifert A, Cotten SR, Xie B. A double burden of exclusion? digital and social exclusion of older adults in times of COVID-19. J Gerontol B Psychol Sci Soc Sci. 17 de febrero de 2021;76(3):e99-103. Doi: 10.1093/geronb/gbaa098 